\title{
Recurrent Cerebral Infarction Caused by Mobile Aortic Arch Thrombus Refractory to Warfarin Therapy
}

\author{
Soo-Kyung Bok, $\mathrm{MD}^{1}$, Young-Jin Lee, $\mathrm{MD}^{2}$, So-Young Ahn, $\mathrm{MD}^{1}$ \\ ${ }^{1}$ Department of Rehabilitation Medicine, Chungnam National University School of Medicine, Daejeon; \\ ${ }^{2}$ Department of Rehabilitation Medicine, Konyang University School of Medicine, Daejeon, Korea
}

The mobile thrombus in the aortic arch is a rare condition, which becomes rarer when associated with cerebral infarction, resulting in disabling complications. Transesophageal echocardiography is useful for detecting the source of thrombi in the heart and aortic arch. Here, we report a case of a patient who suffered from recurrent cerebral infarction four times during the previous 18 months due to mobile thrombus in the aortic arch despite anticoagulant therapy. The thrombus with rocking motion $(9.6$ and $8 \mathrm{~mm}$ ) was laid in the top of the aortic arch, and surgical resection was not allowed due to the location. We began anticoagulation therapy initially with heparin followed by a combination of warfarin, aspirin, and atorvastatin with an international normalized ratio between 2 and 3. The size of the aortic thrombi was significantly decreased by $3 \mathrm{~mm}$ and stabilized after 18 months.

Keywords Aorta, Thrombus, Stroke, Transesohphageal echocardiography

\section{INTRODUCTION}

Aortic thrombus is one of the major causes of arterial embolism. Khatibzadeh et al. [1] found evidence of arterial embolization in $33 \%$ of patients with aortic plaque. In particular, the thrombus in the proximal aortic arch can

Received November 1, 2012; Accepted February 26, 2013 Corresponding author: So-Young Ahn

Department of Rehabilitation Medicine, Chungnam National University Hospital, 282 Munhwa-ro, Jung-gu, Daejeon 301-721, Korea Tel: +82-42-338-2460, Fax: +82-42-338-2461, E-mail: asyoung@cnuh. co.kr

(c) This is an open-access article distributed under the terms of the Creative Commons Attribution Non-Commercial License (http://creativecommons. org/licenses/by-nc/3.0) which permits unrestricted noncommercial use, distribution, and reproduction in any medium, provided the original work is properly cited.

Copyright $(2013$ by Korean Academy of Rehabilitation Medicine cause severe life-threatening stroke as well as limb ischemia.

It is known that more than $85 \%$ of major arterial emboli have their origin in the heart and the remaining $15 \%$ is classified with unknown origins. The advancement of diagnostic tools, such as computed tomography (CT), transesophageal echocardiography (TEE), and magnetic resonance imaging (MRI), has helped improve the diagnostic rates of aortic thrombi. After performing autopsies on 10,671 bodies, Machleder et al. [2] revealed that aortic thrombi were found in 48 cases $(0.45 \%)$, of which 11 cases $(23 \%)$ had evidences of peripheral embolism. Further, Gagliardi et al. [3] found that incidences of peripheral arterial embolism from aortic thrombus increased by $5 \%$. However, the mobile thrombi in the aortic arch are very rare conditions, and it is even rarer for these thrombi to 
evoke embolic cerebral infarction.

We describe four cases in which hypermobile aortic arch thrombus caused embolic cerebral infarction since July 8, 2010.

\section{CASE REPORT}

A 61-year-old male was presented with sudden onset of left upper and lower extremities weakness and aphasia on July 8,2010 . He has a medical history of hypertension and diabetes mellitus and had been taking medication and insulin therapy for three years. Brain MRI revealed newly developed cerebral infarction by occlusion of the right middle cerebral artery (MCA). Chest radiography, echocardiography, and transthoracic echocardiography did not show any abnormalities. Laboratory test for hypercoagulability and lipid profile at admission was normal: low-density lipoprotein $116 \mathrm{mg} / \mathrm{dL}$ (reference, 0 to $130 \mathrm{mg} / \mathrm{dL}$ ), total cholesterol $197 \mathrm{mg} / \mathrm{dL}$ (reference, 0 to $200 \mathrm{mg} / \mathrm{dL}$ ), and homocysteine $7.45 \mu \mathrm{mol} / \mathrm{L}$ (reference, 5.08 to $15.39 \mu \mathrm{mol} / \mathrm{L})$. However, TEE demonstrated echogenic finger-like thrombi with rocking motion (9.6 and $8 \mathrm{~mm}$ ) on the top of the aortic arch (just proximal to the subclavian artery) with minimal atherosclerotic wall. Chest CT showed multiple ulcerations with intramural hematoma (Fig. 1).

We diagnosed embolic cerebral infarction originating from the aortic arch thrombi. The patient was initially treated with intravenous unfractionated heparin for 5 days. Because the location of the aortic arch thrombus did not allow surgical treatment, the patient was treated with $5 \mathrm{mg}$ warfarin, initially, with an internationalized ratio (INR) between 2 and 3. Three days after onset, a rehabilitation program began at the bedside. Training for passive range of motion exercise, rolling and sitting was conducted at bedside. After transferring to the ward, gait training, strengthening exercises of left side muscles and balance training were performed. At the first discharge, 35 days from onset, he had fair grade of weakness in the left proximal upper and lower extremities and poor+ grade in the left distal upper and lower extremities, according to the manual muscle test and grade II spasticity by Modified Ashworth Scale (MAS) in the left upper and lower extremities. He was able to walk indoors independently using a straight cane and plastic ankle-foot orthosis (AFO). The Korean-Modified Barthel Index (KMBI) was 90 points and the Korean-National Institutes of Health Stroke Scale (K-NIHSS) was 5 points. The prescribed warfarin of $4 \mathrm{mg}$ and INR was 2.5 .

On November 8, 2010, the patient was admitted again with progressive weakness of the left extremities and dysarthria. He showed poor grade in the left proximal upper extremities and distal lower extremities. He was not able to walk or sit up by himself. Brain MRI revealed new developed anterior cerebral arterial infarction, and MR angiography conveyed obstruction of distal anterior cerebral artery (Fig. 2). He was treated with increased doses of warfarin by up to $5 \mathrm{mg}$ and comprehensive rehabilitation was initiated. At the second discharge, onset after 25 days, it was examined that the left proximal and distal upper extremities were fair grade, the muscles of the left hip and knee were poor+, ankle was poor, and left extremities had spasticity with grade II by MAS. He was able to walk using a straight cane and plastic AFO under minimal assistance. K-MBI was 68 points and K-NISS was 7 points. He was hospitalized again on April 15, 2011 due to weaknesses in the left extremities and dysarthria, while maintaining $5 \mathrm{mg}$ of warfarin with INR at 2. Left upper and
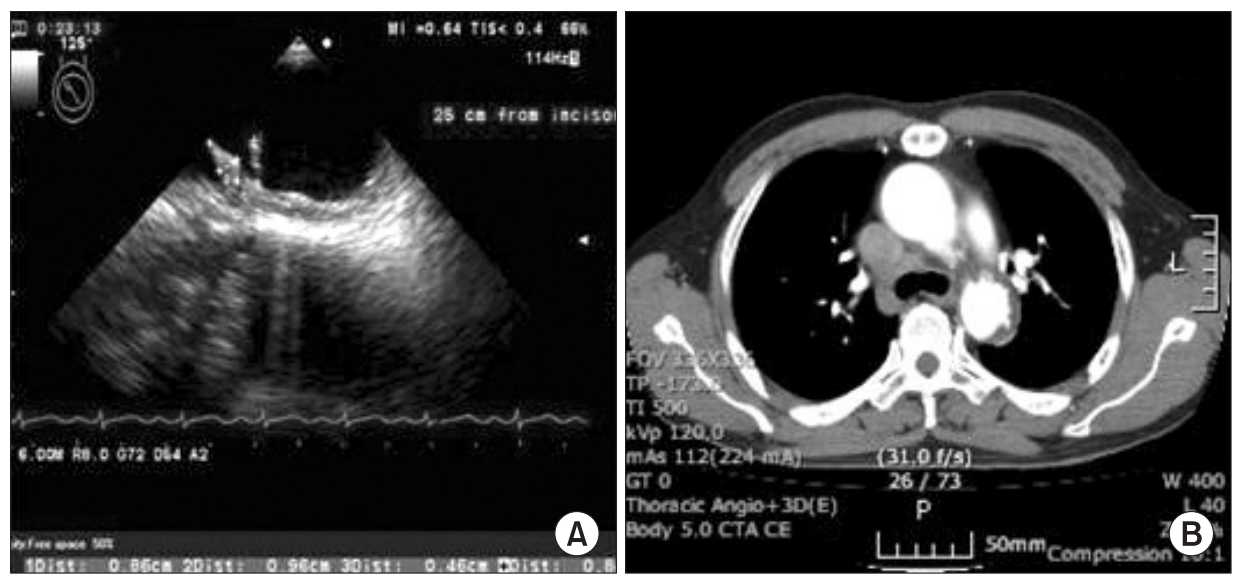

Fig. 1. (A) Transesophageal echocardiography demonstrates fingerlike mobile thrombi of the aortic arch. (B) Multi-detector computed tomography scan demonstrate intramural hematoma. 

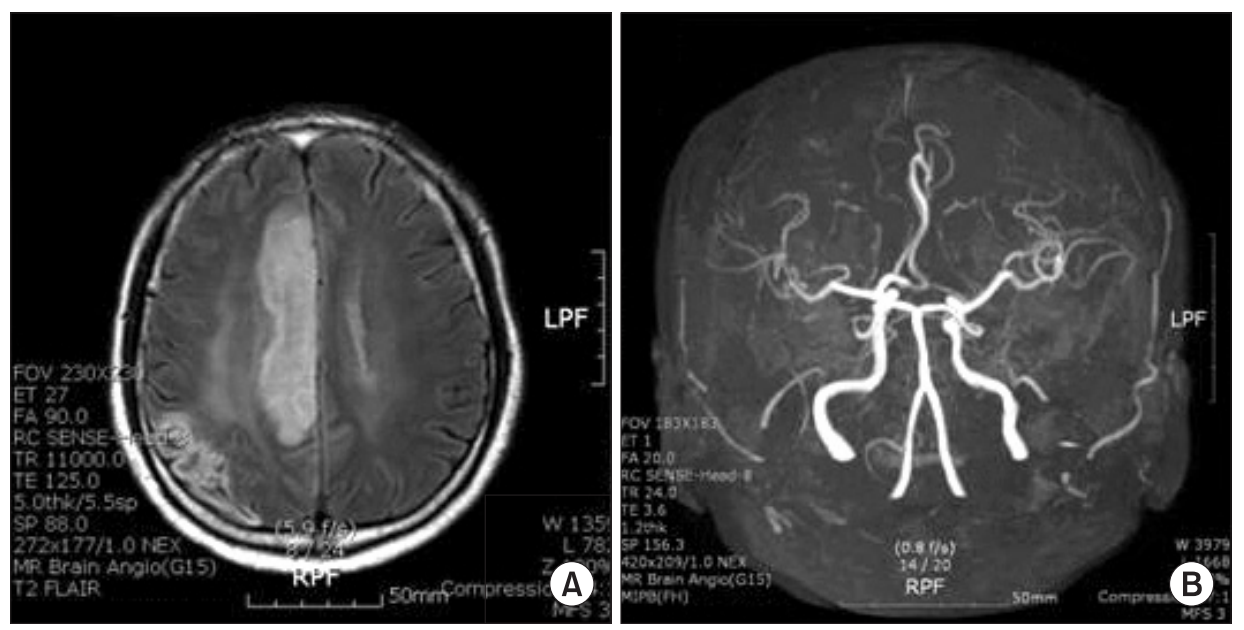

Fig. 2. (A) Brain magnetic resonance imaging, acute infarction on right anterior cerebral arterial territory. (B) Brain magnetic resonance angiography, obstruction of right distal anterior cerebral artery.

proximal lower extremities were poor grade, and ankle dorsiflexor was trace grade. Brain MRI disclosed the recurrence of cerebral infarction of the right MCA territory. He was unable to walk and showed depression symptoms due to stroke recurrence. He and his wife wanted to be discharged after an acute phase treatment, 10 days after onset, and maintain $3 \mathrm{mg}$ of warfarin (INR 3.14) medication at the outpatient clinic. At the third discharge, it was examined that the muscle strength of his left upper and lower extremities was poor grade; he was able to walk 30 $\mathrm{m}$ indoor using a quadricane and AFO under moderate assistance. K-MBI was 60 point. On January 2, 2012, he was unfortunately re-admitted to the stroke unit again with progressive weakness of left extremities and aphasia. He was again diagnosed with right MCA infarction. Brain $\mathrm{MR}$ angiography revealed occlusion in the $\mathrm{Ml}$ area of the right MCA territory. TEE showed one hypermobile aortic arch thrombus of approximately $3 \mathrm{~mm}$ in size at the top of the aortic arch, which was more decreased compared with the last study ( 2 mobile thrombus, 9.6 and $8 \mathrm{~mm}$ ). He was treated with anticoagulation and comprehensive rehabilitation again. Also, he was prescribed $4 \mathrm{mg}$ of warfarin (INR 2.61) and $100 \mathrm{mg}$ of aspirin for additional thrombus control, along with $40 \mathrm{mg}$ of atorvastatin. At the fourth discharge after rehabilitation therapy, 30 days after onset, the strength of muscles in the upper left extremity were trace grade, the muscles of hip were poor, knees were trace, ankles were trace, and the spasticity in the left upper and lower extremities was grade III by MAS. He needed at least minimal assistance for daily activities, including sitting up and standing up. He was able to walk $10 \mathrm{~m}$ with the help of a quadricane and AFO under a moderate degree of assistance. K-MBI was 47 points and K-NIHSS was 8 points.

\section{DISCUSSION}

In this case, recurrent cerebral infarction occurred 4 times during the 18 months due to mobile thrombus in the aortic arch despite anticoagulant therapy.

Aortic thrombus usually occurs accompanying the hypercoagulable state (e.g., antiphospholipid antibody syndrome, circumsporozoite protein antithrombin III deficiency, C protein activation inhibition, homocysteine, factor VIII increase) or the use of steroid or estrogen [4]. It is very rare for aortic wall thrombus to occur due to primary vascular lesions without precedent factors. Aortic thrombus has thus been referred to as the primary aortic thrombus or non-obstructive aortic thrombus in the literature. In pathological studies of aortic walls, the primary aortic thrombi are mostly accompanied with at least minimal arteriosclerotic plaque, which can be an origin of mobile thrombus. However, the pathogenesis of mobile thrombus on the aortic wall is quite different from that of arterial thrombus and has multifactorial thrombogenesis [5]. In the case of this patient, there were no precedent factors either; however, multiple ulcerations with intramural hematoma, which were revealed in the chest CT images, suggested that this aortic thrombus indeed originated from the aorta itself.

Meissner et al. [6] verified that of the 588 patients having TEE in the Stroke Prevention Assessment of Risk in a Community (SPARC) study, aortic plaque was found in $43.7 \%$ of patients, whereas $7.6 \%$ had complex thrombus 
plaque ( $>4 \mathrm{~mm}$ or mobile thrombus). The locations of aortic plaques consisted of $8.4 \%$ ascending aorta (complex thrombus plaque $0.2 \%$ ), $31 \%$ aortic arch (complex thrombus plaque $2.2 \%$ ), and $44.9 \%$ descending aorta (complex thrombus plaque 6.0\%). It is a very rare case that complex thrombus plaque is observed in the aortic $\operatorname{arch}[6]$.

In a study on thrombus plaque and stroke risk by the French Aortic Plaque in Stroke group, the stroke risk was found to have increased as the thrombus plaque thickness increased (odd ratio for thrombus plaque thickness: $1 \mathrm{~mm}, 1.0$; 1 to $3.9 \mathrm{~mm}, 3.9 ; 4 \mathrm{~mm}$ or over, 13.8), indicating that stroke risk significantly increased in patients with thrombus with a thickness of over $4 \mathrm{~mm}$ [7]. There have been a number of case presentations and studies on aortic thrombus. Song et al. [8] reported aortic arch thrombus-induced cerebellar infarction in patients without risk factors of thrombus, while William et al. [9] announced aortic thrombus-induced acute peripheral embolism cases in 20 patients with normal aorta. In the case of this report, aortic arch thrombi were complex thrombus plaques of 9.6 and $8 \mathrm{~mm}$ in size, which engendered embolic cerebral infarction in the territories of anterior and middle cerebral artery four times over a course of 18 months.

Although the treatments and the prognosis of aortic thrombus have been described in a number of literatures, there is still no standardized treatment protocol in place. For drug treatment, anticoagulation agents, such as low-molecular-weight heparin and warfarin, are being recommended. Tsilimparis et al. [10] presented cases in which the administration of warfarin resulted in the decrease of thrombus size and complete remission. For surgical treatment, thrombectomy, endarterectomy, partial arterectomy, aortoplasty, or aorta transplantation can be considered depending on the location of the thrombus in the aorta. In this case, warfarin therapy has been maintained due to the high risk in the surgical treatment of the complex aortic arch thrombus plaque; further, recurrences of cerebral infarction occurred repeatedly. The finding of the decrease of thrombus size from 9.6 to $3 \mathrm{~mm}$ in a TEE in 2012 is considered as the result of anticoagulation therapy. It suggests that the complex thrombus has been stabilized and the risk of cerebral infarction is expected to be reduced. We attempted to prevent the recurrence of stroke through atorvastatin to facilitate the stability of the aortic wall and also through aspirin to prevent additional thrombogenesis.

In conclusion, there exists a potential high risk of embolic infarction when complex aortic plague ( $>4 \mathrm{~mm}$ thick or mobile thrombus) is found. In this case, cerebral infarction recurred 4 times during 18 months of anticoagulation therapy cause by the aortic arch. Anticoagulation with a combination of antiplatelet therapy significantly reduced thrombus size.

\section{CONFLICT OF INTEREST}

No potential conflict of interest relevant to this article was reported.

\section{REFERENCES}

1. Khatibzadeh M, Mitusch R, Stierle U, Gromoll B, Sheikhzadeh A. Aortic atherosclerotic plaques as a source of systemic embolism. J Am Coll Cardiol 1996;27:664-9.

2. Machleder HI, Takiff H, Lois JF, Holburt E. Aortic mural thrombus: an occult source of arterial thromboembolism. J Vasc Surg 1986;4:473-8.

3. Gagliardi JM, Batt M, Khodja RH, Le bas P. Mural thrombus of the aorta. Ann Vasc Surg 1988;2:201-4.

4. Laperche T, Laurian C, Roudaut R, Steg PG. Mobile thromboses of the aortic arch without aortic debris: a transesophageal echocardiographic finding associated with unexplained arterial embolism. The Filiale Echocardiographie de la Societe Francaise de Cardiologie. Circulation 1997;96:288-94.

5. Tunick PA, Kronzon I. Protruding atherosclerotic plaque in the aortic arch of patients with systemic embolization: a new finding seen by transesophageal echocardiography. Am Heart J 1990;120:658-60.

6. Meissner I, Khandheria BK, Sheps SG, Schwartz GL, Wiebers DO, Whisnant JP, et al. Atherosclerosis of the aorta: risk factor, risk marker, or innocent bystander? A prospective population-based transesophageal echocardiography study. J Am Coll Cardiol 2004;44:1018-24.

7. Amarenco P, Cohen A, Tzourio C, Bertrand B, Hommel M, Besson G, et al. Atherosclerotic disease of the aortic arch and the risk of ischemic stroke. N Engl J Med 1994;331:1474-9.

8. Song IW, Hong GR, Cho JH, Jun SY, Son CW, Lee SH, et al. A case of huge thrombus in the aortic arch with 
cerebrovascular embolization. J Cardiovasc Ultrasound 2009;17:148-50.

9. Williams GM, Harrington D, Burdick J, White RI. Mural thrombus of the aorta: an important, frequently neglected cause of large peripheral emboli. Ann Surg 1981;194:737-44.
10. Tsilimparis N, Hanack U, Pisimisis G, Yousefi S, Wintzer C, Ruckert RI. Thrombus in the non-aneurysmal, non-atherosclerotic descending thoracic aorta: an unusual source of arterial embolism. Eur F Vasc Endovasc Surg 2011;41:450-7. 